\title{
Motor-skill learning in older adults - a review of studies on age-related differences
}

\author{
Claudia Voelcker-Rehage
}

Received: 8 August 2007 / Accepted: 4 January 2008 /Published online: 24 January 2008

(C) EGREPA 2008

\begin{abstract}
This paper reviews research on motor-skill learning across the life span with particular emphasis on older age. For this purpose, studies that focus on age-related differences in fine and gross motor skills and studies that analyze the further refinement of known skills as well as learning of unknown motor skills are summarized. The reviewed studies suggest that although motor performance tends to decline in old age, learning capabilities remain intact, and older adults are able to achieve considerable performance gains. The extent to which the learning capability varies with age, however, has to be considered very carefully. While most studies revealed that performance gains in fine motor tasks are diminished in older adults, results for gross-motor-skill learning are more contradictory. Additionally, there is considerable agreement on the finding that age-related learning differences are statistically more robust in complex tasks, whereas in lowcomplexity tasks, the learning of younger and older adults is very similar. This applies to fine and gross motor skills. Relative age differences seem to become enlarged when effortful resources are required for motor performance. Thus, the decline in motor learning that accompanies aging is task specific and not absolute.
\end{abstract}

Keywords Motor skills $\cdot$ Learning $\cdot$ Older adults ·

Life cycle $\cdot$ Human developmental

Motor skills play a crucial role in all phases of the life span. That is, people of all ages perform fundamental motor

\footnotetext{
C. Voelcker-Rehage $(\triangle)$

Jacobs Center on Lifelong Learning and Institutional

Development, Jacobs University Bremen,

Campus Ring 1,

28759 Bremen, Germany

e-mail: c.voelcker-rehage@jacobs-university.de
}

skills, such as walking and grasping, or specific skills, such as hammering a nail, pitching a baseball, or driving a car. The measurement of motor skills is one of the fundamental aspects of measuring human performance. It is well known that aging is accompanied by impairments in sensorimotor [27] as well as cognitive and perceptual functioning [e.g., $41,44,50]$. When people age, they perform complex tasks more slowly and, in some cases, less accurately than they once did. Additionally, they also begin to carry out such tasks in qualitatively different ways [11, 40]. If and how these age-related changes may affect the acquisition of motor skills has not been well investigated. Older adults need to practice and learn new and relearn known motor skills, respectively, as part of new task training, recreational pursuits, or rehabilitation.

During the past centuries, numerous motor-skill-learning studies have been conducted. Most of these studies focus on the processes that affect the learning of a movement task in younger adults. The main focus of motor-skill-learning research was on explaining and describing different variables (e.g., knowledge of results, contextual interference, organization of practice, attentional focus, transfer of training) that affect the planning and control of voluntary movements and the learning of motor skills (for an overview see [1]). Studies that investigate motor-skill learning in older age and across the life span are rare [40].

The aim of this paper is to review studies on age-related differences in motor learning across the life span, with special emphasis on older age, and to endorse a comprehensive perspective on motor development in general and motor plasticity in particular. First, related terms, such as motor learning and motor skills, will be defined, and typical schemes to classify motor skills will be described, followed by a literature review on motor-skill-learning studies. Studies that focus on age-related differences in fine and 
gross motor skills, and studies that analyze the further refinement of known skills as well as learning of unknown motor skills will be summarized, followed by a general discussion. In particular, the importance of life-span studies to judge older adults' learning capabilities will be discussed. A comprehensive understanding of motor-skill learning may need to be especially informed by developments at both extremes of the life course: early childhood and late life.

\section{A life-span developmental approach to assess motor-learning studies}

A general issue for understanding development concerns the interplay between capacities and constraints, as well as gains and losses. Life-span developmental psychology (e.g., [6]) with its emphasis on gains and losses, on the tradeoffs between selection and optimization, and on potentials, i.e., plasticity, of the individual provides an appropriate frame to analyze motor development in older adults $[22,66]$. In life-span psychology, development is not defined in juxtaposition to aging, as is usually the case in models of biological aging. Rather, development and aging are used synonymously and are defined as selective agerelated changes of capacities. The capacities encompass psychological functions (such as self-regulation and working memory), structures (such as personality traits and knowledge systems), and physiological functions (such as fine coordination and endurance). Age-related changes in capacities involve increases, as well as maintenance, transformation, and decreases. In this context, the notion of plasticity, reserve capacity, and developmental reserve capacity $[3,6]$ are used in life-span psychology to denote the difference between realized and maximum potential. In principle, the notion of plasticity also includes changes in the negative direction. A central goal of life-span psychology is to chart the magnitude of plasticity and the extent to which it varies with age. Probably the most direct strategy to examine age changes in the range and limits of plasticity is through active experimental intervention [4], e.g., learning studies.

\section{Motor learning and motor development—motor skills and motor abilities}

When dealing with motor-learning studies, one needs to specify some defining characteristics of related terms, such as motor development, motor learning, motor skills, and motor abilities. The term motor development describes adaptive or functional changes in movement behavior over the life span and the processes or factors that underlie these changes ([14] for a review). It refers to sequential, continuous age-related processes whereby movement behavior changes. The factors within a person that may lead to developmental changes in movement behavior include maturation/aging and experience. Not all changes in movement, however, are development. Thus, motor learning is used to describe relatively permanent changes in the capability for motor skills but related to training and aimed interventions rather than development due to maturation or aging [47]. Motor learning — as it is defined in this paperencompasses the acquisition of new unknown skills as well as relearning and improvement of motor skills acquired in the past. When considering a life-span approach, it is difficult to clearly distinguish motor development from motor learning. Motor development across the life span encompasses learning and development as well as capacities and constraints. A main difference between learning and development is that learning concerns the "microgenesis" and not the "ontogenesis". Therefore, motor learning is not to be related to age. Learning and development, however, are interdependent as the effectiveness of learning sport motor skills depends on the development status, and otherwise, results of learning always enter/influence development. A typical approach to investigate age-related differences in motor learning is to use a mixed within-between-subject design. Learning gains are measured within subjects, age-related differences are measured between subjects.

A movement skill ${ }^{1}$ is an organized and well-coordinated sequence of voluntary body, head, and/or limb movements directed towards a desired outcome. Movements of different body parts must be coordinated to produce a movement skill. Inputs from sensory and cognitive processes are important in determining what an individual chooses to do and how the movements are organized and adjusted. Environmental conditions, task requirements, and persons' characteristics impose spatial and temporal constraints that must be sensed and evaluated to determine what needs to be done [32, 35]. Individuals may respond to a movement situation in different ways, depending on their experience and on their sensory, perceptual, and cognitive functioning. Each of these functions may be viewed as a personal resource that has a developmental course of its own. Dynamic interactions among them determine the course and quality of movement skill development [19, 56]. Besides these so-called general (cognitive-oriented abilities, memory-related processes) and perceptual speed abilities

\footnotetext{
${ }^{1}$ In motor development literature, the term "movement" often refers to the observable act of moving, whereas "motor" refers to the aspects of movement that are not directly observable or the underlying factors that affect movement (e.g., $[19,33])$. In the following, the term motor skill will be used in a broader sense referring to the inner and outer aspects of a movement.
} 
[33], the way people perform and learn motor skills is also influenced by their motor abilities. The term motor ability refers to a general trait or capacity of the individual that is related to the performance of a variety of motor skills [33]. A variety of motor abilities underlies the performance of a motor skill. For example, to serve a tennis ball, the player must perform certain components of that skill, e.g., stance, back swing, forward swing, ball contact, etc. The underlying motor abilities that are involved are, e.g., multilimb coordination, speed of arm movement, aiming, static strength, etc.

\section{Classification of motor skills}

A wide variety of schemes exists to structure and classify the variety of motor skills to identify common characteristics of the various skills. The classification of movement skills may be quite narrow or quite broad. Taxonomies of movement skills are usually derived from the author's interpretation of the relevant body of knowledge and are designed for particular applications. Most schemes are based on experiences and descriptives. Some schemes are more comprehensive, but all fall short of fully capturing the breadth, depth, and scope of human movement [19]. Motor skills may be distinguished with regard to their structure, complexity, and the level of difficulty as well as the familiarity level [e.g., 63]. These classification criteria might have considerable consequences for assumptions regarding the magnitude of plasticity of motor performance.

Structure Motor skills usually refer to a specific class of goal-directed movement patterns such as running, throwing, writing, or speaking [32]. In 1962, Smith and Smith [53] proposed three categories of movement skills: postural, travel or locomotor, and manipulative [8]. Burton and Miller [8] classified movement skills by anatomy (e.g., overhand, underhand, one-hand, two-hand), by object of function (baseball, lacrosse, juggling), and by component (elbow extension, step, trunk rotation). Davis and Burton [16] suggested that there were five basic categories of functional movement tasks: (a) locomotion, (b) locomotion on an object, (c) propulsion, (d) reception, and (e) orientation, which includes object manipulation. Other schemes exist to classify motor skills by developmental categories, such as early movement milestones, fundamental movement skills, specialized movement skills, or other types of categories such as activities of daily living [cf. 8].

More prevalent approaches used in motor learning research are the following: Schmidt [46] differentiates (a) discrete skills (which have a distinct beginning and end), (b) continuous skills (which have no distinct beginning or end; they require repetition of movement patterns), and (c) serial skills (which require various steps or a series of movements to complete the task). Poulton [38] distinguishes (a) open skills (which require the performer to adjust to or regulate an environment containing objects that have spatial and/or temporal qualities) and (b) closed skills (skills whose performance can be planned in advance without expectation of environmental changes or can be made to fit the environment predicted in advance). Cratty [15] based his classification on the amount of muscular involvement necessary to complete/execute a skill. He distinguishes between (a) gross motor skills (require a great deal of muscular involvement - total body and/or multilimb movements) and (b) fine motor skills (very little body movement initiated - usually involves the manipulation of tools or objects while sitting down).

Complexity and difficulty According to Wulf and Shea [70], a skill is defined as complex if it cannot be mastered in a single session, has more than one degree of freedom, and has the potential to be ecologically valid. Wulf and Shea [70] pointed out several differences between simple and complex tasks. They rejected the assumption that principles that are developed through the study of simple skills are generalizable to more complex skills. The authors state: "In fact, research on more complex skills shows that the manipulation of practice variables that result in enhanced learning of simple skills are actually detrimental to the learning of complex skills" (p. 207). The term complexity also refers to the portion of involved subsystems or abilities. Motor-skill learning might be especially influenced by preconditions such as endurance, strength, or other motor abilities such as posture. For example, learning of the high jump is mainly influenced by strength (particularly bounce) and learning of fine motor skills by hand-eye coordination. One may conclude that the higher the complexity level, the higher the task difficulty. The term difficulty level of a task is also defined by the time required to learn the task [63].

Familiarity level The level of familiarity regarding motor skills varies from "not known/learned at all" to "high expertise". In terms of motor-skill learning, motor skills can be considered as unknown/known or as skills that have been acquired in the past and need to be relearned or refined (e.g., fundamental motor skills such as activities of daily living).

Figure 1 draws the complex picture of the classification of motor-skill learning. The classification criteria structure, complexity, difficulty, and familiarity level are shown in the first column. Within each possible task structure (e.g., fine/ gross motor skills), the criteria (complexity, difficulty and 
Fig. 1 Classification scheme of motor skills

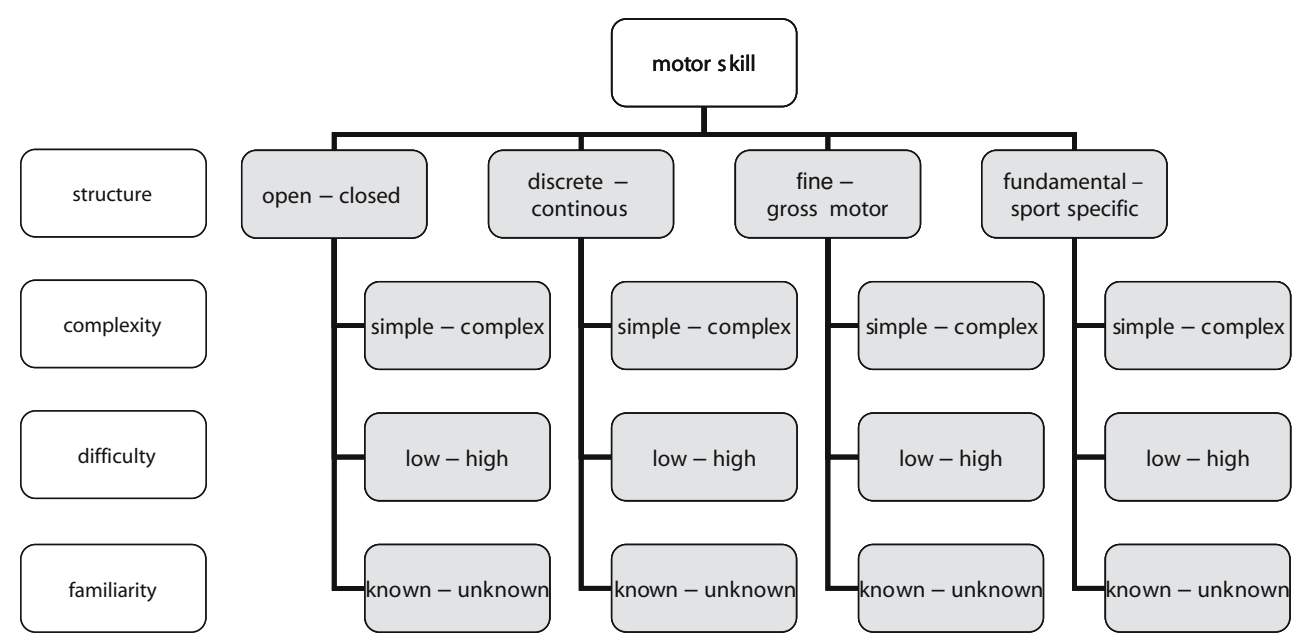

familiarity level) have to be differentiated, leading to high task variability. In the following, the reviewed studies will be classified according to Cratty [15] into fine and gross motor skills. This structure seems to be most appropriate to deal with the manifold motor-skill-learning studies in older adults as the revised studies used very different tasks and research questions to investigate age-related differences in motor-skill learning. Within this structure, studies that focus on age-related differences in motor-skill learning on different complexities and familiarity levels (new learning and refinement of known skills) will be reviewed. First, studies that compare young and older adults will be reported, followed by life-span studies.

\section{Review of motor-skill-learning studies}

The review is based on a comprehensive English and German language literature search of articles up to July 2007 using Medline, PsychInfo, Psychlit, Spofor, and Spolit. A broad search strategy was used: "motor/movement (skill) development", "motor/movement (skill) learning", "plasticity", and "(sport) motor/movement skills" as subject headings (including all subheadings) were combined with "age" and used as keywords. In total, 19 finemotor-skill learning and 6 gross-motor-skill age-related learning studies have been found and will be reviewed.

\section{Motor learning of fine motor skills}

There is a variety of possibilities to conduct fine-motorskill-learning studies. The following fine-motor-skill-learning studies will mainly be organized by their performance outcome measures, such as amount of submovements, force variability, and performance accuracy. As mentioned above, most learning studies conducted on younger adults focus on testing the effects of different variables that may affect the learning of motor skills, such as feedback. In consequence, it is not surprising that some of the aging studies have also been conducted using these questions. In the following, studies that investigated the effect of feedback will be subsumed under the heading of augmented feedback. First, studies that compare young and older adults will be reported, followed by life-span studies.

Submovements Pratt et al. [39] investigated younger (1824 years) and older adults (62-69 years). Subjects practiced rapid aimed limb movements towards a visible target region (100 movements in total). At first, both younger and older adults produced initial ballistic submovements and corrective secondary submovements. Even after extensive practice, older adults did not reduce their submovements whereas younger adults did. However, both groups were able to increase their performance with practice. This suggests that the mechanisms underlying the movements of older adults seem to be qualitatively different from those of younger adults.

This assumption is confirmed in a study by Liao et al. [30]. They reported that older adults (60-69 years) required a longer duration to achieve the same submovement accuracy as younger adults (18-26 years) in a stationary target-acquisition task (manipulation of a onedimensional isotonic control stick; eight blocks of 8-12 trials each). They concluded that a reduced submovement accuracy might be the primary cause of age-related slowing in target acquisition.

Shea et al. [49] investigated age-related differences in the organization of multi-element movement sequences. Young (19-23 years) and old adults (65-68 years) were asked to move a lever as quickly as possible to targets sequentially projected on a tabletop (random and repeated practice blocks (160 targets), random and repeated retention tests). Young and old participants executed the random sequences similarly during acquisition and retention tests 
indicating that there are no age-related differences in (nonspecific) learning characteristics. Age-related differences in the repeated sequences, however, increased over practice with the younger adults performing substantially faster than the older adults. Shea and colleagues concluded that older adults did not organize their movements into subsequences as effectively as younger adults. Older adults were relatively ineffective in using the sequence order information to decrease element duration.

In a study by Seidler [48], young (18-31 years) and old adults (65-80 years) learned different joystick aiming tasks (either making a sequence of actions, adapted to one of two visuomotor rotations, or to an altered gain of display; seven blocks of 24 trials). Older adults revealed slower reaction times than younger adults but (unlike Shea et al. [49]) no deficit in sequence learning. In contrast to the sequencelearning task, older adults exhibited poorer performance and reduced rates of learning for the visuomotor adaptation tasks as compared to younger adults. Seidler concluded that the skill-learning deficits were task specific.

Force variability Voelcker-Rehage and Alberts [64] investigated age-related differences in motor learning using a force modulation task. Young (19-28 years) and old (6775 years) adults used precision grip to perform a variable force-tracking task [sine wave, $5-25 \%$ of the maximal voluntary contraction (MVC); ten blocks of ten trials] with their dominant hand. Results indicated that both groups improved accuracy of force tracking as a result of practice. Younger adults performed the task at a higher level compared to older adults in pre- and post-test conditions. Nonetheless, older adults reached post-test performance levels comparable with younger adults' pre-test performance. Younger adults showed improvements in force control, in force generation and release phases. In contrast, older adults' performance during the force release phases remained quite variable. These data indicate that older adults are impaired in the accurate release of grip force.

Results by Ketcham et al. [26] point in the same direction. Older adults $(M=68$ years, $\mathrm{SD}=6)$ are able to produce forces needed to perform the task (aiming movements on a digitizing tablet, 22 blocks of 12 trials); however, the ability to continuously modulate forces is constrained by the presence of terminal accuracy requirements.

Spirduso et al. [55] investigated younger (18-23 years) and older adults (61-81 years) in tracing a triangle template by controlling the force on spring levers with three different finger combinations (bilateral index finger movement, left index finger and right thumb combination, one-hand pinching movement; 3 days of practice, five trials of each finger combination per day). They found similar practice effects for both age groups, with the exception that the behavioral speed of older adults increased more from the first to the second day of practice, whereas younger adults improved performance in a linear fashion from day 1 to day 3. Older adults, however, were significantly less accurate and required more time in tracing. Thus, younger adults performed on a higher level.

Accuracy In a study by Anshel [2], younger (22-26 years) and older (70-80 years) adults learned a limb repositioning task (20 trials). Younger adults were superior to elders in terms of performance accuracy across trials. Older adults, however, improved to a significantly greater extent than younger adults across trials. Age did not diminish the ability to learn this motor skill but had a negative effect on the performance level.

Breitenstein et al. [7] investigated the accuracy of younger (20-29 years) and older (51-73 years) adults by asking participants to perform two tracking tasks-a simple and a mirror-reversed $(2 \times 3 \mathrm{~min}$ each $)$ - and a serial reaction time task (110 trials). In the simple tracking task (direction of own movement according to movement of tracking stimulus), older adults showed a lower performance level but a similar performance improvement as compared with younger adults. In the mirror-reversed task, younger adults improved performance significantly faster. Apparently, with increased difficulty level, age differences in motor learning become more pronounced.

Augmented feedback Van Dijk et al. [60] investigated young (20-35 years) and old (50-70 years) participants' learning performance in an isometric force-production task (five blocks of 20 trials each, retention test). Participants were provided either with knowledge of results (KR) or kinetic feedback. Age-related differences were not found in the accuracy and consistency of performance and learning. Additionally, an interaction of age with any of the feedback variables was not found, suggesting that the effects of augmented feedback on motor learning are similar in younger and older adults. The authors concluded that a low task complexity might be an explanation for the lack of age-related differences.

Carnahan et al. [13] (cf. also [12]) revealed a similar performance of younger and older adults in a KR study. Participants (older adults 67-79 years, younger adults 20 25 years) were required to learn a computer-key-pressing task in a specified goal time (50 acquisition trials, 10 retention trials). Accuracy or variability differences between the two age groups during retention could not be shown.

Another KR study by Wishart and Lee [68] revealed that younger (19-23 years) and older adults (60-73 years) use $\mathrm{KR}$ in a similar way to learn a motor skill. The authors 
investigated younger and older adults' performance in a three-segment task (90 acquisition trials, retention, transfer, and reacquisition tests). Three different manipulations of relative frequency of information about performance $(100 \%$ KR, 67\% KR faded over trials, 67\% KR faded over segments) were compared. Contradictory to van Dijk et al. [60] and Carnahan et al. [12, 13], but as shown in the "force-studies", results showed age-related differences for movement accuracy and consistency on acquisition and retention tests but not on the transfer test. None of these differences interacted with the frequency of KR manipulations, and there was no effect of the fading schedules. Furthermore, the ability to process KR and the effects of KR on motor learning are similar in young and older adults. Swanson and Lee [57] investigated age differences in a movement timing task. Younger (20-23 years) and older adults (60-82 years) were investigated with regard to the accuracy and consistency of motor performance when KR was provided (90 acquisition trials, 18 retention trials). The researchers found no interaction of age with any of the KRrelated variables. Again, younger adults were more accurate than older adults.

In an augmented feedback study (continuous horizontal flexion-extension movements with a $90^{\circ}$ phase offset between the upper limbs; 50 acquisition tasks each at two consecutive days, $15 \mathrm{~s}$ each) by Swinnen et al. [58], performance levels of the elderly group $(M=72.7$ years, SD $=5.2)$ were lower than that of the group of adolescents $(M=$ 18.8 years, $\mathrm{SD}=1.1$ ) as well as their rate of improvement. Also, an augmented feedback study by Wishart et al. (e.g., [69]) found that older adults learned the task, but they did not reach younger adults' performance levels.

Life-span studies The motor-skill-learning studies described so far, like most of the age-related cognitive learning studies $[4,23,29,45,61,62]$, compare older adults' performances with that of younger adults (mostly students) but do not investigate middle-aged participants or even a wider age range. Recent research from cognitive life-span studies, however, suggests that the age-related changes need to be investigated in more detail. For example, Salthouse [45] showed that many different types of cognitive variables are affected by increased age and that age-related differences appear to begin in early and not in late adulthood. The same might apply to motor-skill learning. Thus, a life-span perspective obtains an estimate of the starting point and the size and the course of potential age-related reductions. A few studies exist that investigated a broader age range. They investigated younger, middleaged, and older adults.

Smith et al. [52] investigated adults between 18 and 95 years in learning a novel visuomotor task (a series of four object-retrieval tasks of increasing difficulty; five times for both right and left hand on each level of difficulty) and motor memory. For data analysis, participants were split into two groups ( 18 to $<62$ years and $62-$ 95 years). The marked slowing of motor speed as well as an increase in performance variance began around 62 years of age. Motor learning was significantly slower in adults over 62 years of age. Motor memory of the newly acquired fine motor skill, however, was preserved in all ages.

Rodrigue et al. [42] investigated the effect of age on the acquisition and long-term retention (5 year follow-up) of a mirror-tracking task (five blocks of five trials each divided on three consecutive days). Participants ranged between 23 and 77 years. Older adults (62-82 years) required more training to reach an asymptote than middle-aged (5456 years) and younger adults (28-36 years). Whereas young and middle-aged adults reached an asymptote by the end of day 2, older adults did not reach asymptotic level until day 3. Effects of aging were revealed and magnified by additional training and thus were greater on the longterm retention test than at baseline.

Durkin et al. [17] tested four groups of adults (19-33, 35-48, 50-63, 66-78 years) over three consecutive days. Subjects were asked to learn a declarative task (verbal memory), a task without a motor component (mirrorreading task, three blocks of 20 trials each on each day), and a task with a motor component (pursuit rotor task, $20 \times$ 20 -s trials per day). The authors showed that the performance increase with practice was hardly affected by age, although there were age-related declines in initial and terminal performance. Both motor and cognitive learning were relatively intact in both groups of older adults. Data suggest that while acquisition deficits in pursuit rotor performance may initially be present in older adults, they are relatively short-term. During the later stage of training, improvement in performance increased in the two older groups, comparable with the younger groups.

\section{Motor learning of gross motor skills}

Comparatively few studies investigated age-related differences in motor learning of gross motor skills [57]. At first, studies that investigated motor tasks on a relatively high level of familiarity and "new learning" studies that compare older and younger adults will be described. Then, the few existing life-span studies regarding motor-skill learning will be discussed.

Hedel and Dietz [24] investigated age-related differences in the acquisition and performance of a precision locomotor task (walking on a treadmill and stepping over obstacles) with full and restricted vision. Younger adults (20-27 years) and older adults (59-81 years) improved performance with 
practice, both with full and restricted vision. However, only younger adults regained optimal foot clearance with practice under restricted vision. Hedel and Dietz concluded that this might indicate that older adults rely more on visual control when acquiring and performing a precision locomotor task. This result is in line with the reported fine motor-skill-learning studies (cf. [7]) that learning gains and performances are comparable in more simple tasks and magnify with task complexity (e.g., more difficult visuomotor coordination).

Kirchner and Schaller [28] investigated "new" learning of the task "balance and turn a stick" in a sample of middleaged and older adults (balancing on a divided bar and at the same time throwing a stick around $180^{\circ} ; 5$ weeks, once per week). They investigated older adults being split into three groups (50-59, 60-69, over 70 years). Learning improves linearly with practice in all three age groups. The 5059 year olds revealed highest pre-test performance, but also lowest performance increases with practice. On the other hand, the learning curves of the groups 60-69 years and over 70 years were a little steeper, although the oldest participants performed on a slightly lower level. This leads to reduced age-related differences in the post-test as compared to the pre-test. Nevertheless, the older groups (60-69 years and $>70$ years) did not reach the performance level of the 50-59 year olds.

Tunney et al. [59] investigated age-related differences in learning the use of a standard walker to get into the passenger side of a four-door sedan using a specific procedure. Younger (20-35 years) and older adults (6193 years) had no prior experience using a walker. They practiced the task for $20 \mathrm{~min}$. Younger adults scored significantly higher than older adults on the final trial of the training session and on a 48-h follow-up test. Performance decline over $48 \mathrm{~h}$ was significantly higher for older than for younger adults, indicating that older adults do not acquire and maintain a functional motor skill as accurately as younger adults.

Perrot and Bertsch [37] investigated younger (2030 years) and older adults (61-75 years) in a balljuggling-task (12 sessions of $20 \mathrm{~min}$ each). They revealed age-related differences in the rate of learning, i.e., acquisition by the younger adults was significantly faster than that by the older adults. They also showed that motor execution for the older adults seemed to require more psychomotor ability and was dependent on cognitive control.

Gershon [20] conducted the first study that investigated the learning of a complex motor skill across the life span. He investigated motor-skill learning in a one-hand juggling task with two balls (ten sets of five trails each per hand). The 136 participants between 6 and 79 years were divided into eight age groups (6-9..70-79 years). Gershon found the highest juggling performances after training in the age group 30-39 years. Young children (6-9 years) and old adults (70-79 years) performed on the lowest level. The results indicate that the learning of a formerly unknown skill-i.e., juggling with two balls in one hand-is possible in all age groups. Old participants' results can be compared to those of young children. Performance of male participants decreased rapidly from the age group 30-39 to 4049 years, remained more or less stable until the age group 60-69 years, and decreased again until the age of 70 79 years. Female participants' performance decreased linearly between $30-39$ and $70-79$ years. This study illustrates that performance decrements start early in middle age and not in old age.

Voelcker-Rehage and Willimczik [65] (cf. also [67]) conducted a life-span study and analyzed motor-skill learning of two different tasks within the same sample. They investigated motor plasticity in motor-skill learning using a juggling and a lacrosse catching task. Their first interest was in the development of juggling and lacrosse performance within the group of older adults. Their second objective was to compare the older adults' performances to the performances of children, youth, and younger adults. This comparison seems to be necessary to obtain an estimate of the size of age-related reductions in plasticity [67]. The sample size of the study was $n=1206$, including ages from 6 to 89 years (602 male and 604 female). For data analysis, participants were divided into age-groups in 5 -year intervals. None of the participants had any prior experience with juggling and lacrosse. Thus, the emphasis of the study was on "new" learning and not on maintenance or improvement of motor skills acquired in the past. Juggling performance was acquired at three times of the learning process; the authors investigated the extent to which the performance before semantic instruction (pre-test 1), after semantic instruction (pre-test 2), and the potential to further refine a complex motor task by training (post-test) is preserved in older adults. Lacrosse performance was investigated at two times, after semantic instruction and after practice. Participants were asked to learn two kinds of juggling (first, juggling with three scarves and after that, juggling with three balls; six sessions, 15 min each) and lacrosse catching (six sessions, $35 \mathrm{~min}$ each).

The main findings for juggling performance were: (1) Older adults show a high potential to acquire and further refine the complex motor task juggling. (2) No statistically significant decrease in motor plasticity between the ages of 60 and 79 years could be observed. (3) Older adults' performance was comparable to the performance of children in the age group 10-14 years and of adults between 25 and 59 years. Performance remained stable in middle age. Only youths and young adults (between 15 and 29 years) performed on a higher level and showed a peak in performance (cf. Fig. 2). The main findings for lacrosse 
Fig. 2 Juggling performance across the life span [raw data points $(M)$ and polynomial trend lines]. Results are shown for baseline performance (pre-test 1), performance after semantic instruction (pre-test 2), and performance after six practice sessions (post-test) [65]

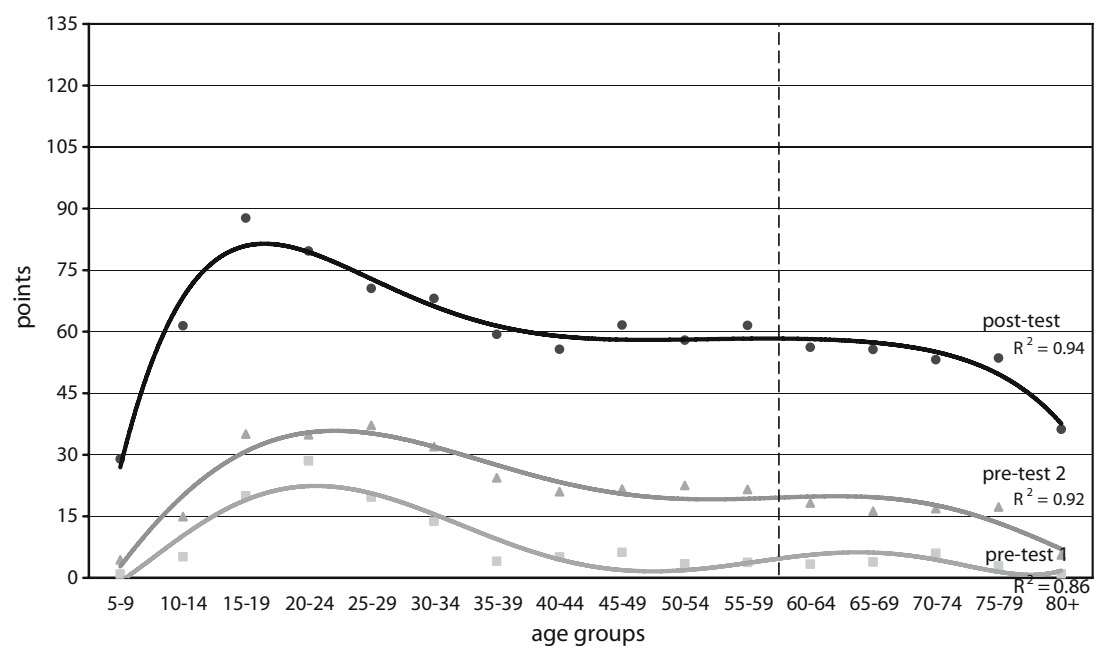

performance were: (1) Lacrosse performance decreased from about 30 years on until old age (cf. Fig. 3). (2) Overall, older adults (between 55-59 and 70-74 years) reached lacrosse performance levels comparable with performance of 10-14 year old children. Participants over 80 years and children between 5 and 9 years of age performed significantly lower than all other age groups. The age group over 80 years, however, showed highest performance improvements with practice. In comparison with the juggling performance, older participants revealed a more dramatic decrease in lacrosse performance with increasing age and a lower performance improvement due to practice, particularly from age 70-74 onwards.

\section{Discussion}

The starting point of this paper was to review studies on motor learning across the life span, particularly in older age. For this purpose, studies that focus on age-related differences in fine and gross motor skills with different complexity and familiarity levels were summarized. The findings suggest that older adults are able to achieve considerable gains in performance. The extent to which plasticity varies with age, however, has to be considered very carefully. Learning differences as well as performance differences seem to be related to the structure of the task, the task complexity, the task difficulty, and the familiarity level.

A common result of most studies is, as shown for studies focusing on motor functioning (not learning), that there is a general tendency that the performance level is lower for older adults as compared with younger adults $[2,7,26,30$, $39,48,49,55,57,58,64,68,69]$. In addition, regardless of learning gains, older adults function on a lower level.

Most studies revealed that performance gains in fine motor skills are diminished in older adults. Thus, performance differences between younger and older adults even increased with practice [26, 39, 49, 58]. Results of gross motor skill learning are contradictory. While Kirchner and
Fig. 3 Lacrosse performance across the life span [raw data points $(M)$ and polynomial trend lines]. Results are shown for performance after semantic instruction (pre-test) and performance after six practice sessions (post-test)

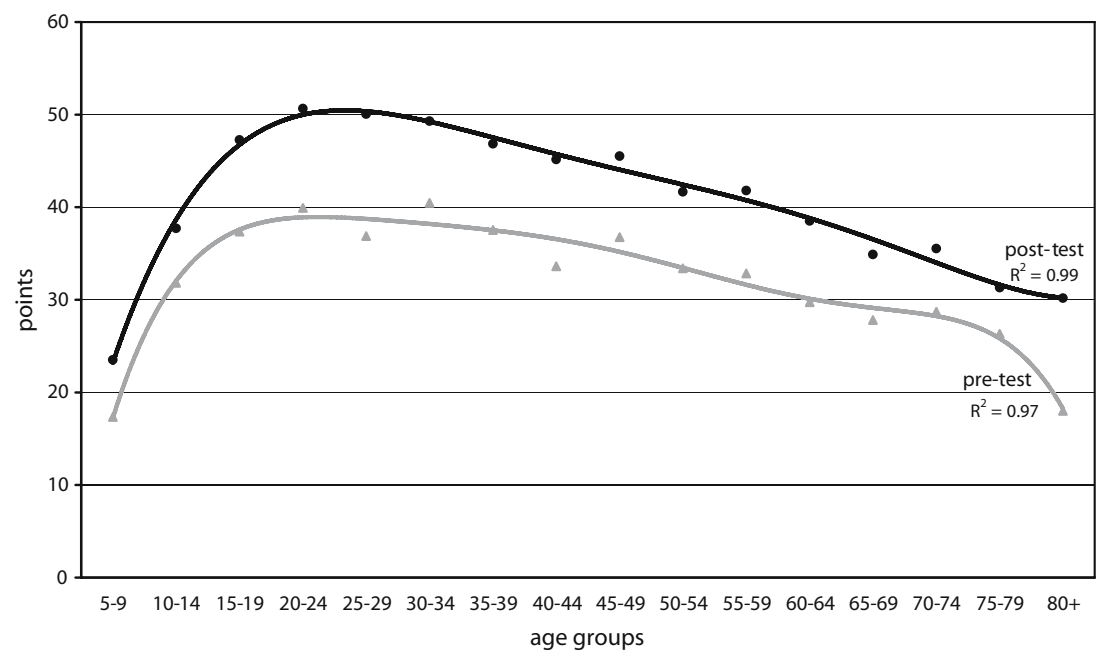


Schaller [28] revealed the highest improvement in the oldest age group, Gershon [20], Perrot and Bertsch [37], and Tunney et al. [59] showed an advantage for the younger adults, and Voelcker-Rehage and Willimczik [65] as well as Hedel and Dietz [24] showed contradictory results with regard to the motor skills.

The studies by Carnahan et al. [12, 13], van Dijk et al. [60], and Spirduso et al. [55] on fine-motor-skill learning revealed a similar learning gain for older and younger adults. One explanation for the lack of age difference is that the tasks may not have been complex enough to identify age difference. Motor control research suggests that as the task difficulty increases, the differences between young and old adults also increase [31, 54].

In the study by Voelcker-Rehage and Willimczik [65], older participants revealed a lower initial performance in the lacrosse task with increasing age and a lower performance improvement due to practice, particularly from age 70-74 onwards, as compared with the juggling performance. The different results in juggling and lacrosse might also point to specific task characteristics such as complexity and difficulty level. The lacrosse task might be more influenced by physical preconditions as compared to the juggling task. The lacrosse catching task required the participants to react to different flight curves and flight directions of the ball. The participants' agility, movement speed, and muscular preconditions might be very important for successful performance on the task. The lacrosse task might be considered more complex. Juggling also required the participant to react to the scarves and/or balls but in a more predictable manner. The physical fitness and motor abilities coupled with the mechanical requirements of the task might greatly influence the ability to move with control, skill, and confidence. Hence, age-related performance differences are more visible in complex as compared to simple tasks. This holds true for both fine and gross motor skills. Apparently, relative age differences become enlarged when effortful resources are required for motor performance. Thus, the decline in motor functioning and learning that accompanies aging is task specific and not absolute.

It is striking that visuomotor performance seems to be adversely affected by age. For instance, Seidler [48] showed specific skill-learning deficits in older adults. Performance differences particularly occurred in a visuomotor adaptation task. Additionally, Breitenstein et al. [7] showed that older adults had problems to perform a visuomotor mirror-reversed task. In a gross-motor-task study, Hedel and Dietz [24] showed that older adults rely more on visual control when acquiring and performing a precision locomotor task. Whether these results are due to the visuomotor restrictions/requirements of the tasks or due to the high complexity level of the tasks remains uncertain.
The level of familiarity seems to be another task characteristic that causes important age-related differences in motor-skill learning. The studies that focus on the learning of fine motor tasks use skills with a rather high familiarity level (put in a new context), for example, hand movements (aiming, sequencing, force modulation). It might be the case that in the fine motor tasks that investigated the refinement of known skills (e.g., hand movements or aiming) rather than "new" learning, initial performance is closer to peak performance. Cognitive "testing the limit" studies revealed an enlargement of age differences when performance limits are approximated by training [4, 62]. One other explanation for the different learning gains of older adults in fine and gross motor tasks might be the performance outcome measures: In the tasks where all factors could simultaneously affect performance, the age-related difference in performance might be less visible than in tasks using more 'precise' or detailed measures. Whereas performance gains in the gross motor tasks are mostly recorded using outcome measures such as points, number of successful trials, etc., performance in fine motor tasks is usually measured by more kinetic or biophysical measures such as movement or force variability or submovements. This might be one reason for the higher amount of studies showing age-related differences in fine motor tasks as compared to gross motor tasks. In gross motor tasks, older adults might be more capable to activate reserve capacities, compensate for motor and cognitive weakness and, in turn, show learning gains comparable to younger adults.

In general, diminished motor-skill-learning gains are interpreted as a substantial age-related performance loss in older adults and a reduction in cognitive or motor plasticity, respectively. Causes of the performance decreases in older age are hypothesized to be neuro-physiological and physiological changes [e.g., 50]. Mechanisms discussed in this context are, for example - on a central level — reduced nerve conduction speed and, in turn, reduced reaction speed, increased lateralization, and diminished inhibition processes, or - on a peripheral level-diminished tactile sensitivity (e.g., [9, 21, 41, 43]).

Age-related neurodegenerative and neurochemical changes are thought to underlie the decline in motor and cognitive performance, but compensatory processes in cortical and subcortical functions (e.g., changed activation patterns, de-differentiation [9], de-lateralization [10, 36]) may allow maintenance of performance (and probably learning) level in older adults. In brain-imaging studies, activation seen early in practice involves generic attentional and control areas-prefrontal cortex, anterior cingulate cortex, and posterior parietal cortex are the main areas considered to perform the scaffolding role (together with changes seen in task-specific areas) [25]. Particularly, the 
prefrontal and mediofrontal cortex and the frontostriatal network are shown to demonstrate highest age-related decline ([9] for an overview). This might be one possible explanation for the slower and/or lower learning gains of older adults.

Individual differences in motor plasticity in old age might be strongly associated with sensory (hearing and vision) and cognitive functioning (memory, speed, fluency, knowledge). It is shown in cognitive studies that participants who had a greater loss in perceptual speed showed a lower maximum performance level and less learning gains [5]. Results suggest that aging-induced biological factors are a prominent source of individual differences in cognitive and, in turn, motor plasticity. Motor and cognitive plasticity cannot be stated as being independent from each other. In particular, the early learning phase has been proven to be mainly influenced by cognitive processes $[25$, 34] to understand the task and prepare strategies.

Studies presented within this review indicate that regardless of performance decreases, considerable learning improvements are possible in older age. The life-span perspective makes it possible to obtain an estimate of the size of age-related reductions in plasticity, and it underlines the high amount of remaining motor plasticity in older age. A typical comparison of younger (mostly students) versus older adults - as done in most cognitive and motor studies - often underestimates the performances of the older adults, particularly in learning of new gross motor skills. The life-span study results indicate that the reduction in motor plasticity occurs not particularly in older age but also in young and middle age (after a peak in youth and younger adulthood). For example, the grossmotor-skill study by Gershon [20] illustrates that performance decrements start early in middle age and not in old age. Same is shown in the study by Voelcker-Rehage and Wilimczik [65]. However, results differ with regard to the task characteristics. Whereas juggling performance decreased between 19 and 35 years and remained stable until older age (69 years), lacrosse performance nearly linearly decreased from the age of 29 years onwards. In all studies, there is a substantial decrease in the oldest old (around 80 years). Only one study revealed that performance decreases start in older age, from the age of 62 onwards; motor learning was significantly slower in adults over 62 years of age [52]. Furthermore, one life-span study revealed no age-related differences with regard to motor learning across adulthood [17].

A limitation of studies on age-related differences is that the age-comparison is based on a mixed cross-sectional design. Although performance changes due to practice are measured longitudinally in a pre-post-test design, the age comparisons are limited to age-related averages and evidence about long-term changes at the individual level is not available. Particularly in older age, individuals vary considerably in their individual performance level [54] and probably also in their performance gains due to learning. Additionally, cross-sectional studies that cover a wide age span-whether it be a young-old comparison, or a comparison of multiple age groups across the life spanhave the problem that they can be threatened by cohort effects. Thus, age-related differences shown in the described studies may not only represent age-related differences but also reflect cohort-related preconditions.

A further limitation of aging studies is the sample selectivity. In the case of aging populations, the generalizability of results can be impaired in the ways that the average level of functional competence and/or learning is overestimated if individuals with lower levels of functioning are less likely to participate in a study than individuals with higher levels of functioning. Furthermore, it is undeniable that the incidence of disability in older groups progressively increases. One of the ways other than age that two or more age groups in cross sectional research could be different is the incidence of disabilities that could impact performance [18]. In general, one can assume that all studies described above appoint comprehensive screenings before the start of the study to eliminate participants with health-related or cognitive impairments that could potentially influence the outcome of the study.

If we focus on motor-skill learning in older age, however, we need to take into consideration that this is a very broad age range covering about 30 years. Cognitive change in the very old, the so-called fourth age, proves special features and constraints: Sensory limitations, slower speed of processing, limits to independence, and motor limitations are common characteristics of both the start and the end of our lives. Until now, performance gains in very old age have been investigated using only cognitive tasks. Singer et al. [51] investigated performance gains after mnemonic instructions in a sample of 75- to 101-year-old subjects (eight sessions of instruction and training in a performance-enhancing mnemonic technique: Method of Loci). It was found that memory plasticity with the Method of Loci is still preserved in very old age, although to a limited degree. At the same time, the comparison of the acquisition functions of young and very old participants during the adaptive training period revealed an enlargement of age differences: Apparently, very old adults had a reduced ability to optimize their performance after instruction.

The evidence for age-related reduction of motor plasticity founded on the basis of the comparison of young and old participants and life-span studies could not be demonstrated within all the reviewed studies. The findings show that although the performance of motor skills is greatly affected by age, acquisition of a skill is relatively 
unaffected by age. Adult capacities for extensive learning and change represent the open end of development.

Acknowledgments I would like to thank Klaus Willimczik for his critical review and insightful comments on the manuscript and for making it possible to conduct the MODALIS life-span study. Further, I would like to thank Ben Godde and our human performance group for their helpful comments on the manuscript.

\section{References}

1. Adams JA (1987) Historical review and appraisal of research on the learning, retention, and transfer of human motor skills. Psychol Bull 10:41-74

2. Anshel MH (1978) Effect of aging on acquisition and short-term retention of a motor skill. Percept Mot Skills 47:993-994

3. Baltes PB (1987) Theoretical propositions of life-span developmental psychology: On the dynamics between growth and decline. Dev Psychol 23:611-626

4. Baltes PB, Kliegl R (1992) Further testing of limits of cognitive plasticity: negative age differences in a mnemonic skill are robust. Dev Psychol 28:121-125

5. Baltes PB, Lindenberger U (1997) Emergence of a powerful connection between sensory and cognitive functions across the adult life span: a new window to the study of cognitive aging. Psychol Aging 12:12-21

6. Baltes PB, Lindenberger U, Staudinger UM (1998) Life-span theory in developmental psychology. In: Lerner RM (ed) Handbook of child development. Theoretical models of human development. Wiley, New York 1029-1143

7. Breitenstein C, Daum I, Schugens MM (1996) Altersunterschiede beim motorischen Lernen [Age differences in motor learning]. Z Gerontopsychol Psychiatr 1:33-41

8. Burton AW, Miller DE (1998) Movement skill assessment. Human Kinetics, Champaign, IL, USA

9. Cabeza R (2001) Functional neuroimaging of cognitive aging. In: Cabeza R, Kingstone A (eds) Handbook of functional neuroimaging of cognition. MIT Press, Cambridge, MS, USA, pp 331377

10. Cabeza R (2002) Hemispheric asymmetry reduction in older adults: The HAROLD model. Psychol Aging 17:85-100

11. Cabeza R, Daselaar SM, Dolcos F, Prince SE, Budde M, Nyberg L (2004) Task-independent and task-specific age effects on brain activity during working memory, visual attention and episodic retrieval. Cereb Cortex 14:364-375

12. Carnahan H, Vandervoort AA, Swanson LR (1993) The influence of aging on motor skill learning. In: Stelmach GE, Hömberg V (eds) Sensorimotor impairment in the elderly. Kluwer, Netherlands, pp 41-56

13. Carnahan H, Vandervoort AA, Swanson LR (1996) The influence of summary knowledge of results and aging on motor learning. Res Q Exerc Sport 67:280-287

14. Clark JE, Whitall J (1989) What is motor development: The lessons of history. Quest 41:183-202

15. Cratty BJ (1964) Movement behavior and motor learning. Lea \& Febiger, Philadelphia

16. Davis WE, Burton AW (1991) Ecological task analysis: Translating movement behavior theory into practice. Adapted Phys Act Q 8:154-177

17. Durkin M, Prescott L, Furchtgott E, Cantor J, Powell DA (1995) Performance but not acquisition of skill learning is severely impaired in the elderly. Arch Gerontol Geriatr 20:167-183
18. Gabell A, Nayak USL (1984) The effect of age on variability in gait. J Gerontol 39:662-666

19. Gallahue DL, Ozmun JC (2002) Understanding motor development. McGraw-Hill, New York, NY, USA

20. Gershon EJ (1978) A study of age and sex differences in the acquisition of a complex motor skill. Rev Educ Phys 18:362-372

21. Grady CL (2000) Functional brain imaging and age-related changes in cognition. Biol Psychol 54:259-281

22. Haywood KM, Getchell N (2001) Life span motor development, 3rd edn. Human Kinetics, Champaign, IL, USA

23. Head D, Raz N, Gunning-Dixon F, Williamson A, Acker JD (2002) Age-related differences in the course of cognitive skill acquisition: the role of regional cortical shrinkage and cognitive resources. Psychol Aging 17:72-84

24. Hedel HJA, Dietz V (2004) The influence of age on learning a locomotor task. Clin Neurophysiol 115:2134-2143

25. Kelly AMC, Garavan H (2005) Human funtional neuroimaging of brain changes associated with practice. Cereb Cortex 15:10891102

26. Ketcham CJ, Seidler RD, Van Gemmert AWA, Stelmach GE (2002) Age-related kinematic differences as influenced by task difficulty, target size, and movement applitude. J Gerontology, Psychol Sci 57B:P54-P64

27. Ketcham CJ, Stelmach GE (2001) Age-related declines in motor control. In: Birren JE, Schaie KW (eds) Handbook of the psychology of aging. Academic, San Diego, CA, USA, pp 313-348

28. Kirchner G, Schaller HJ (1996) Motorisches Lernen im Alter: Grundlagen und Anwendungsperspektiven [Motor learning in older adults: Foundation and perspectives]. Meyer \& Meyer, Aachen, Germany

29. Li SC, Lindenberger U, Hommel B, Aschersleben G, Prinz W, Baltes PB (2004) Transformations in the couplings among intellectual abilities and constituent cognitive processes across the lifespan. Psychol Sci 15:155-163

30. Liao M-J, Jagacinski RJ, Greenberg N (1997) Quantifying the performance limitations of older and younger adults in a target acquisition task. J Exp Psychol Hum Percept Perform 23:16441664

31. Light KE, Spirduso WW (1990) Effects of adult aging on the movement complexity factor of response programming. J Gerontol B Psychol Sci 45B:P107-P109

32. Magill RA (1993) Motor learning: Concepts and applications, 4th edn. Brown \& Benchmark, Dubuque, IA, USA

33. Magill RA (2004) Motor learning and control, 7th edn. Mc Graw Hill, New York

34. Milton JG, Small SS, Solodkin A (2004) On the road to automatic: dynamic aspects in the development of expertise. J Clin Neurophysiol 21:134-143

35. Newell KM (1986) Constraints on the development of coordination. In: Wade MG, Whiting HTA (eds) Motor development in children: Aspects of coordination and control. Martin Nijhoff, Amsterdam, pp 341-361

36. Nyberg L, Sandblom J, Jones S, Neely AS, Petersson KM, Ingvar M, Bäckman L (2003) Neural correlates of training-related memory improvement in adulthood and aging. Proc Natl Acad Sci 100:13728-13733

37. Perrot A, Bertsch J (2007) Role of age in relation between two kinds of abilities and performance acquisition of new motor skill. Percept Mot Skills 104:91-101

38. Poulton EC (1957) On prediction in skilled movement. Psychol Bull 54:467-478

39. Pratt J, Chasteen AL, Abrams RA (1994) Rapid aimed limb movements: age differences and practice effects in component submovements. Psychol Aging 9:325-334

40. Rabbitt P (1997) Ageing and human skill: a 40th anniversary. Ergonomics 40:962-981 
41. Raz N (2000) Aging of the brain and its impact on cognitive performance: integration of structural and functional findings. In: Craik FIM, Salthouse TA (eds) The handbook of aging and cognition. Erlbaum, Mahwah, NJ, USA, pp 1-90

42. Rodrigue KM, Kennedy KM, Raz N (2005) Aging and longitudinal change in perceptual-motor skill acquisition in healthy adults. J Gerontol B Psychol Sci 60B:P174-P181

43. Salthouse TA (1985) Speed of behavior and its implications for cognition. In: Birren JE, Schaie KW (eds) Handbook of the psychology of aging. Van Nostrand Reinhold, New York, pp 400 426

44. Salthouse TA (1985) A theory of cognitive aging. North-Holland, Amsterdam

45. Salthouse TA (2004) What and when of cognitive aging. Am Psychol Soc 13:140-144

46. Schmidt RA (1975) Motor skills. Harper and Row, New York

47. Schmidt RA, Lee TD (1999) Motor control and learning, 3rd edn. Human Kinetics, Champaign, IL, USA

48. Seidler RD (2006) Differential effects of age on sequence learning and sensorimotor adaptation. Brain Res Bull 70:337-346

49. Shea CH, Park J-H, Braden HW (2006) Age-related effects in sequential motor learning. Phys Ther $86: 478-488$

50. Shumway-Cook A, Woollacott M (2000) Attentional demands and postural control: the effect of sensory context. J Gerontol A Med Sci 55A:M10-M16

51. Singer T, Lindenberger U, Baltes PB (2003) Plasticity of memory for new learning in very old age: a story of major loss. Psychol Aging 18:306-317

52. Smith CD, Walton A, Loveland AD, Umberger GH, Kryscio RJ, Gash DM (2005) Memories that last in old age: motor skill learning and memory preservation. Neurobiol Aging 26:883-890

53. Smith KU, Smith WH (1962) Perception and motion. Saunders, Philadelphia

54. Spirduso WW, Francis KL, MacRae PG (2005) Physical dimensions of aging. Human Kinetics, Champaign, IL, USA

55. Spirduso WW, Smith A, Choi JH (1993) Age and practice effects on force control of the thumb and index finger in precision pinching and bilateral coordination. In: Stelmach GE, Hömberg V (eds) Sensorimotor impairment in the elderly. Kluwer, Netherlands, pp 393-412

56. Sudgen D, Keogh J (1990) Problems in movement skill development. University of South Caroliner Press, Columbia

57. Swanson LR, Lee TD (1992) Effects of aging and schedules of knowledge of results on motor learning. J Gerontol B Psychol Sci 47B:P406-411
58. Swinnen SP, Verschueren SMP, Bogaerts H, Dounskaia N, Lee TD, Stelmach GE, Serrien DJ (1998) Age-related deficits in motor learning and differences in feedback processing during the production of a bimanual coordination pattern. Cogn Neuropsychol $15: 439-466$

59. Tunney N, Taylor LF, Gaddy M, Rosenfeld A, OPearce N, Tamanini J, Treby A (2003) Aging and motor learning of a functional motor task. Phys Occup Ther Geriatr 21:1-16

60. van Dijk H, Mulder T, Hermens HJ (2007) Effects of age and content of augmented feedback on learning an isometric forceproduction task. Exp Aging Res 33:341-353

61. Verhaeghen P, Marcoen A (1996) On the mechanism of plasticity in young and older adults after instruction in the method of loci: Evidence for an amplification model. Psychol Aging 11:164-178

62. Verhaeghen P, Marcoen A, Goossens L (1992) Improving memory performance in the aged through mnemonic training: a metaanalytic study. Psychol Aging 7:242-251

63. Voelcker C, Wiertz O, Willimczik K (1999) Möglichkeiten und Grenzen einer Re-Interpretation von Untersuchungen zum motorischen Lernen [Lifespan motor development - a re-interpretation of investigations on motor learning]. Psychol Sport 6:90-101

64. Voelcker-Rehage C, Alberts JL (2005) Age-related changes in grasping force modulation. Exp Brain Res 166:61-70

65. Voelcker-Rehage C, Willimczik K (2006) Motor plasticity in a juggling task in older adults - a developmental study. Age Ageing $35: 422-427$

66. Willimczik K, Conzelmann A (1999) Motorische Entwicklung in der Lebensspanne-Kernannahmen und Leitorientierungen [Motor development through the life span-main assumptions and guidelines]. Psychol Sport 6:60-70

67. Willimczik K, Voelcker-Rehage C, Wiertz O (2006) Sportmotorische Entwicklung über die Lebensspanne-Empirische Befunde $\mathrm{zu}$ einem theoretischen Konzept (Motor development across the lifespan-empirical results of a theoretical concept). Z Sportpsychol 13:10-22

68. Wishart LR, Lee TD (1997) Effects of aging and reduced relative frequency of knowledge of results on learning a motor skill. Percept Mot Skills 84:1107-1122

69. Wishart LR, Lee TD, Cunningham SJ, Murdoch JE (2002) Agerelated differences and the role of augmented visual feedback in learning a bimanual coordination pattern. Acta Psychol 110:247263

70. Wulf G, Shea CH (2002) Principles derived from the study of simple skills do not generalize to complex skill learning. Psychol Bull Rev 9:185-211 